\title{
Expression of the caspase-8 gene in neuroblastoma cells is regulated through an essential interferon- sensitive response element (ISRE)
}

\author{
Cell Death and Differentiation (2004) 11, 131-134. doi:10.1038/ \\ sj.cdd. 4401327 \\ Published online 7 November 2003
}

\section{Dear Editor,}

It is well known that caspase-8 is an 'initiator' caspase acting at the top of the apoptotic cascade. ${ }^{1-3}$ Specific ligand engagement by death receptors of the TNF-R superfamily or CD95/FAS induces receptor aggregation and recruitment of the Fas-associated death domain and caspase-8 molecules. ${ }^{4,5}$ Thus, caspase- 8 becomes activated and further activates, by enzymatic cleavage, the downstream 'effector' caspases. ${ }^{6,7}$ The silencing of caspase-8 gene was observed in several tumors, where it was related to resistance to deathinducing ligands, such as TNF $\alpha$, TRAIL or CD95/FAS. ${ }^{8,9}$ It has been proposed that in aggressive human neuroblastomas (NB), silencing of caspase-8 depends on methylation of a regulatory region at the boundary between caspase-8 exon-3 and intron-3. ${ }^{10}$

Interferon (IFN)- $\gamma$ has been shown to sensitize several malignant cells to apoptosis ${ }^{11-13}$ through upregulation of caspase-8 expression. ${ }^{12,13}$ Intriguingly, IFN- $\gamma$ also induced caspase-8 expression in tumor cells having a hypermethylated caspase-8 gene, without affecting DNA methylation. ${ }^{12}$ In addition, we recently showed that the transcriptional inactivation of caspase- 8 gene is independent of CpG methylation of this gene in a fraction of primary NB. ${ }^{14}$ These observations led us to the first identification of a $1.2 \mathrm{~kb}$ DNA element, located $5^{\prime}$ to the first exon of caspase- 8 gene, which acts as a promoter in NB cell lines that express caspase-8, but not in NB cells that lack constitutive expression of this gene. ${ }^{14}$

Here, we studied the mechanisms of caspase-8 gene regulation by IFN- $\gamma$ in the SH-SY-5Y NB cells, which showed virtually no constitutive expression of caspase-8. The treatment of SH-SY-5Y cells with IFN- $\gamma$ for $48 \mathrm{~h}$ induced a strong caspase-8 expression (Figure 1a). Since several IFN- $\gamma$ sensitive genes are regulated through the STAT-1/IRF-1 pathway, ${ }^{15-19}$ we studied the activation of this signal transduction pathway in these cells. IFN- $\gamma$ signaling first induces phosphorylation of JAK-1 and -2, which in turn activate STAT- 1 by phosphorylation. pSTAT- $1 \alpha$ homodimers directly induce the transcription of specific genes, including the transcription factor IRF-1, ${ }^{17}$ which further activates other IFN- $\gamma$-sensitive genes. ${ }^{18,19}$ Induction of the phosphorylated form of STAT- 1 was detected in SH-SY-5Y cells by a specific antiserum to p-tyr 701 of STAT-1, $20 \mathrm{~min}$ after IFN- $\gamma$ stimulation (Figure 1a). Moreover, the transcription factor IRF-1 was strongly upregulated $24 \mathrm{~h}$ after stimulus, indicating that the STAT-1/IRF-1 pathway is active in these NB cells, and could be involved in caspase-8 gene transcriptional control. IRF-2, an IFN- $\gamma$-related factor regulating IRF-1 binding to the ISRE and/or its activity, was constitutively expressed in $\mathrm{SH}$ SY-5Y NB cells and also appeared to be upregulated by IFN- $\gamma$. Finally, the upstream factor USF-1, involved in the transcriptional coactivation of several IFN- $\gamma$-dependent genes, was also constitutively expressed.

Prior to promoter regulation studies, we investigated the caspase-8 transcription initiation site(s) by RACE analysis, using exon-5-specific primers (data not shown). Sequencing of RACE products indicated that caspase- 8 transcripts mostly originated at the position -20 of the caspase-8 cDNA reference sequence (NM_001228), although the finding of fragments of different length suggested the possible existence of other transcription initiation sites. Indeed, a different caspase-8 transcription initiation start site was recently reported by primer extension studies in human hepatoma cell lines. ${ }^{20}$ We then tested the possible regulation of the promoter $\mathrm{P}-1161 /+16$ (Figure $1 \mathrm{~b}$ ) by IFN- $\gamma$. Treatment with IFN- $\gamma$ induced CAT expression in P-1161/ +16/CAT-transfected SH-SY-5Y cells (Figure 1c), indicating that the P-1161/ + 16 fragment contains IFN- $\gamma$-inducible regulatory elements. Two GAS elements (at positions -787 and -188 ) potentially capable of binding PSTAT-1 and two ISRE (at -567 and 32) were found in this region (Figure 1b). An additional ISRE at -38 was partially overlapped with the ISRE-32. In addition, two E-boxes, potentially capable of binding the USF-1 transcription factor, were also present at positions -95 and -54 .

To better characterize the elements required for promoter activity, we further performed deletion studies. Fragments of $167,368,600$ and $843 \mathrm{bp}$, respectively, named $\mathrm{P}-151 /+16$, $352 /+16,-584 /+16$ and $-827+16$, having the same $3^{\prime}$ end were generated by PCR amplification, sequenced and cloned into the CAT reporter plasmid (Figure 1b). When transfected into SH-SY-5Y cells, all these DNA fragments showed no promoter activity, although CAT expression was upregulated after IFN- $\gamma$ treatment (Figure 1d). Interestingly, the promoter activity of $\mathrm{P}-151 /+16$ was strongly upregulated, indicating that $\mathrm{P}-151 /+16$ represents a 'minimal' IFN- $\gamma$-sensitive promoter. Moreover, the ISRE at position -38 and/or -32 should play a pivotal role in IFN- $\gamma$-induced transcriptional regulation, since no other potential IRF-1 or STAT-1-binding sites were present in $\mathrm{P}-151 /+16$.

On the basis of computer analysis, ISRE-32 is more similar to the canonical IRF-1-binding site than ISRE-38. To study the functional role of the ISRE at position -32 in the promoter context, we generated P-1161/ +16 and P-584/ +16/CAT constructs, where only the ISRE-32 site was disrupted by a 
point mutation (Figure 1e). Both these mutated constructs were inactive as promoters in IFN- $\gamma$-treated SH-SY-5Y, confirming the critical role of this site in the transcriptional regulation of caspase- 8 by IFN- $\gamma$ (Figure 1e).

To prove that the minimal promoter fragment is actually capable of binding IRF-1, IRF-2 and USF-1 transcription factors, we used a $5^{\prime}$ biotin-labeled $\mathrm{P}-151 /$ + 16 DNA fragment immobilized to streptavidin-magnetic beads. Nuclear extracts from IFN- $\gamma$-treated or -untreated SH-SY-5Y cells were allowed to react with immobilized promoter $\mathrm{P}-151 /+16$, and bound proteins were analyzed by Western blotting using IRF-1-, IRF2- or USF-1-specific antibodies. As shown in Figure 1f, a specific $48 \mathrm{kDa}$ band corresponding to IRF-1 was clearly present in the eluted fraction from IFN- $\gamma$-treated $\mathrm{SH}-\mathrm{SY}-5 \mathrm{Y}$ cells extracts. IRF-1 binding was specifically competed by the addition of an excess of free P-151/+16 DNA. IRF-2 and USF-1 were also capable of binding to the minimal promoter sequence, using nuclear extracts from both untreated or IFN$\gamma$-treated SH-SY-5Y cell lines. The use of an immobilized DNA deleted of the ISRE (P-151/-40) showed no IRF-1 or IRF-2 in the eluted fraction from the same nuclear extracts (not shown).

Our data indicate that IFN- $\gamma$ is capable of upregulating the transcriptional activity of a caspase-8 promoter, which we have identified in the genomic region $5^{\prime}$ flanking caspase- 8 and exon-1. Although this region contains two STAT-1 and three IRF-1 potential binding sites, which may play a role in caspase- 8 regulation in vivo, both promoter deletion analysis

a
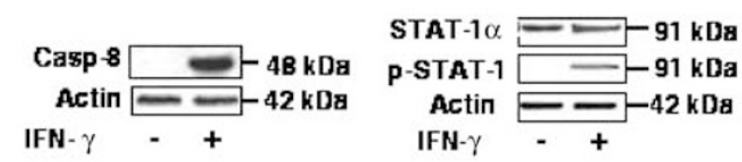

b

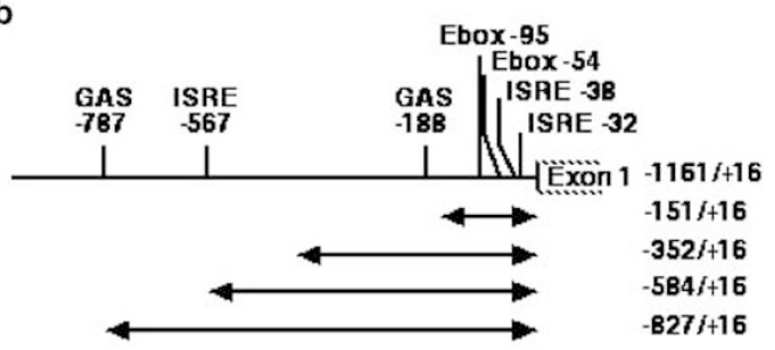

d

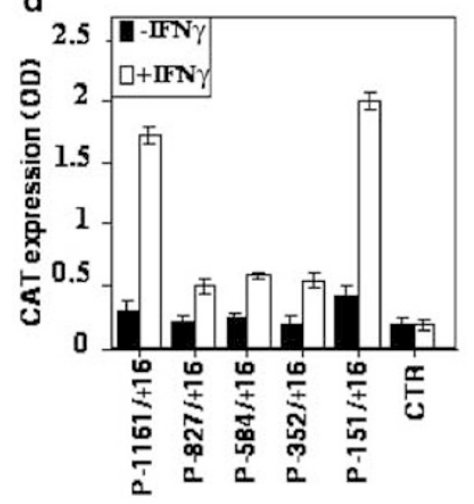

f

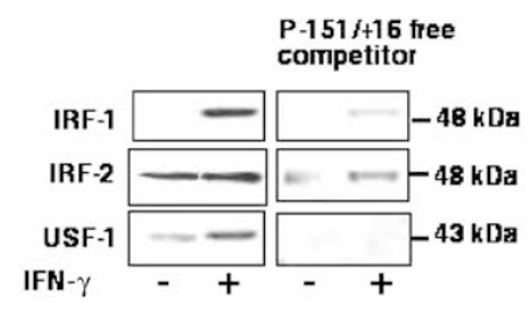

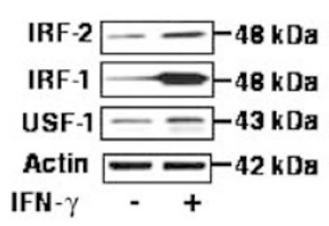

C

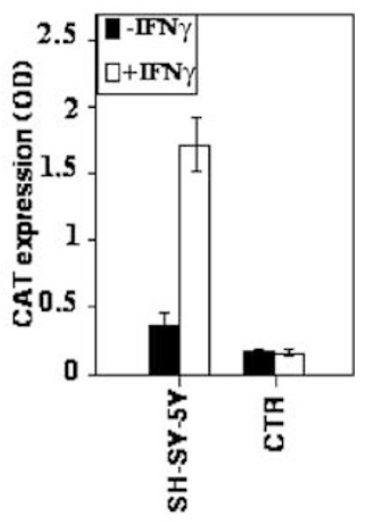

e

ISRE-32
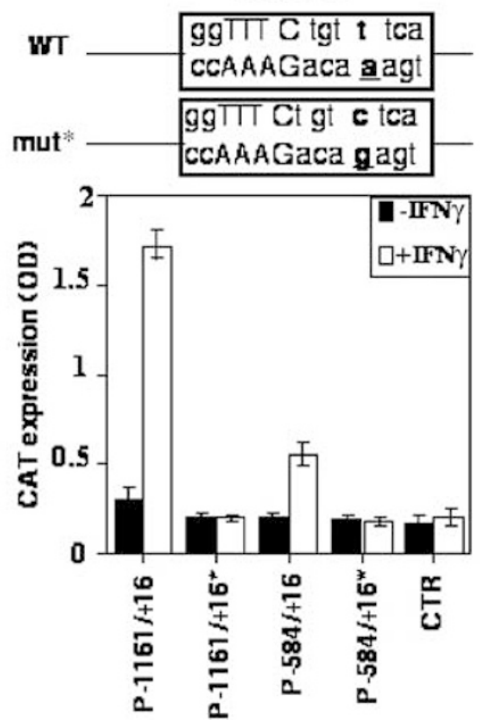
and site-specific mutagenesis showed that the ISRE-32, proximal to exon-1, is essential for the IFN- $\gamma$-mediated promoter activation. However, this finding does not deny the possibility that the other IRF-1- and STAT-1-binding sites may play a role in vivo in a more complex nuclear environment. Previous reports pointed out the importance of STAT-1 in the regulation of caspase- 8 gene by IFN- $\gamma$, since STAT-1deficient $\mathrm{T}$ cells ${ }^{21}$ or U3A cells ${ }^{12}$ are resistant to apoptosis due to their lack of caspase-8 expression, and could not be induced to express caspase- 8 by IFN- $\gamma$. A recent report indicated that a GAS site within a caspase-8 promoter, similar to that identified by us, ${ }^{14}$ plays an important role in caspase- 8 gene regulation. ${ }^{13}$ In view of the complexity of the caspase- 8 genomic region, and the existence of several caspase-8 isoforms, ${ }^{4,5,22,23}$ the presence of other caspase-8 promoter(s), possibly regulating inducible and/or constitutive expression cannot be definitively ruled out. ${ }^{10}$

Multiple promoters have been reported for other genes, such as the MHC-class II-transcriptional transactivator CIITA. $^{16}$ In the IFN- $\gamma$-sensitive CIITA P-IV promoter, pSTAT-1 homodimers play a direct role in transcriptional regulation by binding to GAS sequence(s) in concert with IRF1 , IRF-2 and USF-1. ${ }^{24}$ Our findings that the activity of the caspase-8 minimal promoter $(P-151 /+16)$, lacking a GAS sequence, is upregulated by IFN- $\gamma$ suggest that STAT- 1 plays an important indirect role in the regulation of caspase-8, through the induction of IRF-1. DNA-protein interaction studies indeed showed that IRF-1 is capable of binding to the minimal promoter $\mathrm{P}-151 /+16$. Similarly, in other IFN- $\gamma$ sensitive genes, such as the catepsin-S, ${ }^{17}$ a single IRF-1binding site located upstream the transcription start site was found to be critical for transcriptional activation. The important role of IRF-1 in caspase-8 regulation was also evidenced by a previous study showing that the gene transfer of IRF-1 is sufficient to restore the expression of caspase-8 and sensitivity to apoptosis in NB cells lacking constitutive caspase-8 expression. ${ }^{17}$

The finding that not only IRF-1 but also IRF-2 binds to the minimal promoter sequence, points to a role of IRF-2 in the regulation of caspase-8 gene expression. However, the constitutive expression of IRF-2 in cells lacking the expression of caspase- 8 indicates that IRF-2 alone is unable to activate caspase- 8 promoter. In other promoters, it has been proposed that IRF-2 may act either as a negative regulator of the IRF-1mediated promoter activation, ${ }^{25}$ or, on in contrast, as a cooperative factor acting in concert with IRF-1. ${ }^{24}$

The finding that a point mutation in the ISRE-32 completely abrogates IFN- $\gamma$-inducible promoter activity is not a unique feature of this promoter, since other studies reported similar findings on different promoters. It has been proposed that mutations in these regulatory regions may alter the conformation of the transcription factor/chromatin complex, thus preventing the coordinate interaction with other factors required for the formation of a functional enhanceosome or transcriptosome. ${ }^{26,27}$

The ubiquitous transcriptional activator USF-1 is known to bind upstream E-boxes in other IFN- $\gamma$-sensitive promoters, and to play a relevant role in transcriptional activation through pSTAT-1 or IRF-1 ${ }^{28,29}$ interaction with their specific binding sites. Thus, the detection of two E-boxes at positions -95 and -54 , upstream the critical ISRE-32, and the detection of USF1 binding to this caspase-8 promoter region, strongly support a role of USF-1 in the transcriptional regulation of caspase-8.

These data may provide a better understanding of the molecular mechanisms involved in caspase-8 gene transcriptional regulation and in the related cellular sensitivity to apoptotic stimuli.

\section{Acknowledgements}

B Banelli is a fellow of the Fondazione Italiana per la Ricerca sul Cancro (FIRC). M Croce is a fellow of the Fondazione Italiana per la Lotta al Neuroblastoma. This work was supported by grants from the Fondazione Italiana per la Lotta al Neuroblastoma, from the Associazione Italiana per la Ricerca sul Cancro (AIRC) and from the Italian Health Ministry.

\section{Casciano $^{1,4}$, A De Ambrosis ${ }^{2,4}$, M Croce $^{2,4}$, G Pagnan $^{3}$, A Di Vinci ${ }^{1}$, G Allemanni ${ }^{1}, B$ Banelli ${ }^{1}, M$ Ponzoni ${ }^{3}, M$ Romani $^{1}$ and S Ferrini ${ }^{* 2}$}

Figure 1 (a) IFN- $\gamma$ induces STAT-1 phosphorylation, and IRF-1 and caspase-8 expression in SH-SY-5Y cells, while USF-1 is constitutively expressed, and IRF-2 is upregulated. STAT and pSTAT- 1 were tested at $20 \mathrm{~min}$, IRF- 1 and IRF- 2 at $24 \mathrm{~h}$, and caspase- 8 at $48 \mathrm{~h}$ from IFN- $\gamma$ stimulation $(1000 \mathrm{IU} / \mathrm{ml})$. A total of $2 \times 10^{6} \mathrm{cells}$, either untreated or treated with IFN- $\gamma$, were lysed in lysis buffer ( $10 \mathrm{mM}$ Tris-HCl pH 7.5, $150 \mathrm{mM} \mathrm{NaCl}, 1 \mathrm{mM} \mathrm{EDTA}, 0.1 \%$ SDS, $1 \%$ NP-40 and a cocktail of protease inhibitors) for $30 \mathrm{~min}$ on ice. For the detection of pSTAT-1, cells were washed in PBS containing $400 \mathrm{M} \mathrm{Na}_{3} \mathrm{VO}_{4}$ and lysed in a buffer containing $1 \mathrm{mM} \mathrm{Na}{ }^{3} \mathrm{VO}$. Equal amounts of proteins of each sample were run on $12 \%$ SDS-polyacrylamide gels and blotted onto nitrocellulose membranes. After blocking, membranes were stained using rabbit polyconal anti-IRF-1, anti-IRF-2, anti-STAT-1 anti-USF-1, anti- $\beta$-actin (Santa Cruz Technology, CA, USA), anti-p-STAT-1 (New England Biolabs, MA, USA) or monoclonal anti-Caspase-8 (MBL, Nagoya, Japan) followed by anti-mouse or anti-rabbit IgG horseradish peroxidase-conjugated antibodies. Membranes were developed by the ECL system (Amersham, UK). (b) Schematic representation of the caspase-8 promoter region, of relevant transcription factor-binding sites and of promoter deletion constructs. Identification of the putative transcription-binding sites within the promoter sequence was performed using the Matlnspector software (http://www.genomatrix.de/) (release 6.1, matrix 3.1.1). This construct contains nucleotides $1524605-1525781$ from the reference genomic sequence NT_005370.10 and corresponds to nucleotides P-1161/ +16 of the caspase- 8 gene. (c) Promoter activity of a DNA fragment $(P-1161 /+16) 5^{\prime}$ flanking caspase- 8 exon- 1 is upregulated by IFN- $\gamma$ in caspase-8-negative SH-SY-5Y NB cells. Data are expressed as CAT expression detected on cell lysates by a CAT-ELISA assay (Roche, Milano, Italy). The cloning of a $1.2 \mathrm{~kb}$ caspase-8 promoter into the pBL-CAT3 reporter vector has been described previously. ${ }^{14} \mathrm{NB}$ cells were transfected with the constructs or with the empty vector by the FuGENE system (Roche). Transfection efficiency (22-30\%) was verified using the pEGFPN1 plasmid (Clontech, Beckton Dickinson Italia $\mathrm{SpA}$, Milano, Italy) and FACS analysis. To determine the effect of IFN- $\gamma$ cells were cultured in a medium containing IFN- $\gamma$ after transfection. (d) Promoter activity of different deleted DNA fragments in SH-SY-5Y NB cell lines is upregulated by IFN- $\gamma$. The following promoter deletion fragments were generated by PCR, sequenced and cloned in the pBL-CAT3 reporter vector: $-1161 /+16,-827 /+16,-584 /+16,-352 /+16,-151 /+16$. (e) Site-specific mutagenesis of the ISRE-32 abrogates the promoter activity. The promoter activity of WT and point mutated $\left({ }^{*}\right)$ P-1161/ + 16 or P-584/ + 16 DNA fragments was tested in IFN- $\gamma$-stimulated SH-SY-5Y cells. The IRF-1 site at position -32/-19 was destroyed by site-directed mutagenesis of the core sequence. The authenticity of the constructs was verified by sequencing. (f) IRF-1, IRF-2 and USF-1 from SH-SY-5Y nuclear extracts binding to the caspase-8 promoter fragment $5^{\prime}$ to exon- 1 in vitro. Nuclear extracts were prepared from either IFN- $\gamma$ treated or -untreated SH-SY-5Y cells. ${ }^{30}$ Nuclear extracts $(150 \mathrm{~g})$ were allowed to bind with biotinylated P-151/ + 16 DNA immobilized to steptavidin-coated magnetic beads (Dynal-Oxoid, Italy), in the presence or absence of a $20 \mathrm{M}$ excess of free competitor P-151/ + 16 DNA, for 30 min at RT. Bound material was eluted in a $400 \mathrm{mM}$ $\mathrm{NaCl}$ HEPES buffer, and analyzed by Western blotting using IRF-1-, IRF-2- or USF-1-specific antibodies, as described above 
1 Laboratory of Tumor Genetics, IST-Genova Largo Rosanna Benzi 10, Genova 16132, Italy

2 Laboratory of Immuno-pharmacology, Istituto Nazionale per la Ricerca sul Cancro (IST-Genova), Largo Rosanna Benzi 10, Genova 16132, Italy

3 Differentiation Therapy Unit, Laboratory of Oncology, Istituto G Gaslini, Largo G Gaslini 5, Genova 16147, Italy

4 These authors contributed equally to this work

* Corresponding author: S Ferrini, Laboratory of Immuno-pharmacology, Istituto Nazionale per la Ricerca sul Cancro, Largo Rosanna Benzi 10, Genova 16132, Italy. Tel: + 39-0105737-372; Fax: + 39-0105737-374;

E-mail: silvano.ferrini@istge.it

1. Tamm I et al. (2001) Lancet Oncol. 2: 33-42

2. Fischer U et al. (2003) Cell Death Differ. 10: 76-100

3. Nunez $G$ et al. (1998) Oncogene 17: 3237-3245

4. Muzio M et al. (1996) Cell 85: 817-827

5. Boldin MP et al. (1996) Cell 85: 803-815

6. LeBlanc HN and Ashkenazi A (2003) Cell Death Differ. 10:66-75

7. Peter ME and Krammer PH (2003) Cell Death Differ. 10: 26-35

8. Eggert A et al. (2001) Cancer Res. 61: 1314-1319

9. Fulda $\mathrm{S}$ et al. (2001) Oncogene 20: 5865-5877
10. Teitz T et al. (2000) Nat. Med. 6: 529-535

11. Annicchiarico-Petruzzelli M (2001) Med. Pediatr. Oncol. 36: 115-117

12. Fulda S and Debatin KM (2002) Oncogene 21: 2295-2308

13. Yang $X$ et al. (2003) Cancer Res. 63: 1122-1129

14. Banelli $B$ et al. (2002) Nat. Med. 8: 1333-1335

15. Ramana CV et al. (2000) Oncogene 19: 2619-2627

16. Muhlethaler-Mottet A et al. (1997) EMBO J. 16: 2851-2860

17. Storm van's Gravesande K et al. (2002) J. Immunol. 168: 4488-4494

18. Tanaka N et al. (1993) Mol. Cell. Biol. 13: 4531-4538

19. Decker T et al. (1997) J. Interferon Cytokine Res. 17: 121-134

20. Liedtke $C$ et al. (2003) J. Biol. Chem. 278: 27593-27604

21. Refaeli $Y$ et al. (2002) J. Exp. Med. 196: 999-1005

22. Fernandes-Alnemri T et al. (1996) Proc. Natl. Acad. Sci. USA 93: 7464-7469

23. Himeji $D$ et al. (2002) Blood 99: 4070-4078

24. Xi H and Blanck G (2003) Mol. Immunol. 39: 677-684

25. Blanco JC et al. (2000) J. Exp. Med. 191: 2131-2144

26. Falvo JV et al. (1995) Cell 83: 1101-1111

27. Thanos D and Maniatis T (1995) Cell 83: 1091-1100

28. Muhlethaler-Mottet A et al. (1998) Immunity 8: 157-166

29. Gongora $C$ et al. (2000) Nucleic Acids Res. 28: 2333-2341

30. Boyd KE et al. (1998) Proc. Natl. Acad. Sci. USA 95: 13887-13892

\section{Corrigendum}

\section{Subcellular localisation of Cdc25A determines cell fate}

\author{
C Leisser, G Rosenberger, S Maler, G Fuhrmann, M Grusch, \\ S Strasser, S Huettenbrenner, S Fassl, D Polgar, S Krieger, \\ C Cerni, R Hofer-Warbinek, R deMartin and G Krupitza \\ Cell Death and Differentiation (2004) 11, 134. doi:10.1038/ \\ sj.cdd. 4401370
}

Correction to: Cell Death and Differentiation advanced online publication 5 September 2003; doi:10.1038/ sj.cdd. 4401318

Since the advanced online publication, the authors have identified an error. The spelling of $R$ deMartin is incorrect, the correct spelling should be: $\mathrm{R}$ de Martin. 